\title{
Study on the Connection Between the Set Transient in RRAMs and the Progressive Breakdown of Thin Oxides
}

\author{
Fernando Leonel Aguirre ${ }^{\circledR}$, Member, IEEE, Alberto Rodriguez-Fernandez ${ }^{(}$, \\ Sebastián Matías Pazos ${ }^{(}$, Member, IEEE, Jordi Suñé $^{\mathbb{D}}$, Fellow, IEEE, \\ Enrique Miranda $^{\circledR}$, Senior Member, IEEE, and \\ Félix Palumbo ${ }^{\circledR}$, Member, IEEE
}

\begin{abstract}
In this paper, the transition rate (TR) from the high-resistance state to the low-resistance state of a $\mathrm{HfO}_{2}$-based resistive random access memory (RRAM) is investigated. The TR is statistically characterized by applying constant voltage stresses in the range from 0.45 to $0.65 \mathrm{~V}$. It is found that TR follows a voltage dependence which closely resembles the one exhibited by metalinsulator-semiconductor / metal-insulator-metal structures when subjected to constant voltage stress, but with remarkably different fitting parameters. This result suggests a common underlying mechanism in both evolutionary behaviors. Furthermore, the investigation provides additional evidence supporting the micro-structural changes in the oxide after the forming step as well as the role played by the atomic species during the SET event.
\end{abstract}

Index Terms-High-k, progressive oxide breakdown, resistive random access memory (RRAM), resistive switching (RS).

\section{INTRODUCTION}

$\mathbf{R}$ ESISTIVE random access memory (RRAM) is attracting considerable interest as a promising future technology for scalable memory [1] and neuromorphic computing

Manuscript received May 15, 2019; revised May 31, 2019; accepted June 5, 2019. This work was supported in part by the MINCyT under Contract PICT2013/1210, Contract PICT2016/0579, and Contract PME2015-0196, in part by CONICET under Project PIP$11220130100077 \mathrm{CO}$, in part by UTN.BA under Project PID-UTN EIUTIBA4395TC3, Project CCUTIBA4764TC, Project MATUNBA4936, and Project CCUTNBA5182, and in part by the European institutions. The work of J. Suñé and E. Miranda was supported by the Spanish Ministerio de Ciencia, Innovación y Universidades and the ECSEL EU Joint Undertaking through the WAKeMeUP 783176 Project. The review of this paper was arranged by Editor J. Kang. (Corresponding author: Félix Palumbo.)

F. L. Aguirre, S. M. Pazos, and F. Palumbo are with the Unidad de Investigación y Desarrollo de las Ingenierías, Facultad Regional Buenos Aires, Universidad Tecnológica Nacional, Buenos Aires C1179AAQ, Argentina, and also with the Consejo Nacional de Investigaciones Científicas y Técnicas, Buenos Aires C1425FQB, Argentina (e-mail: aguirref@ieee.org; spazos@frba.utn.edu.ar; felix.palumbo@ conicet.gov.ar).

A. Rodriguez-Fernandez, J. Suñé, and E. Miranda are with the Departament d'Enginyeria Electrònica, Universitat Autènoma de Barcelona, 08193 Cerdanyola del Vallès, Spain (e-mail: alberto.rodriguez @uab.cat; jordi.sune@uab.cat; enrique.miranda@uab.cat).

Color versions of one or more of the figures in this paper are available online at http://ieeexplore.ieee.org.

Digital Object Identifier 10.1109/TED.2019.2922555 systems [2]. RRAM relies on the formation and dissolution of a nanoscale conductive filament (CF) spanning an insulating layer, typically a binary transition-metal oxide, such as $\mathrm{NiO}$ [3], $\mathrm{TiO}_{x}$ [4], $\mathrm{HfO}_{x}$ [5], and $\mathrm{TaO}_{x}$ [6], among others. The CF is initially generated by a forming operation, namely, a controlled dielectric breakdown (BD) of the insulating layer using an appropriate current compliance level [7]. A small gap in the localized CF, which is responsible for the low-resistance state (LRS) in the memory device, is then opened through a RESET operation causing the transition from the LRS to the high-resistance state (HRS) and closed with a SET operation (HRS to LRS transition). SET/RESET operations can be achieved via electrical pulses of the same or opposite polarities (unipolar/bipolar switching modes, respectively). The resistive switching (RS) mechanism has been widely studied in the last years [1]-[12], and many authors claim that the SET/RESET processes are described through ion migration induced by the local electric field and the temperature increase associated with Joule heating [10]. Ion migration is modeled by diffusion and drift components, where both rely on hopping mechanisms. Ions move along an energy landscape of potential wells, which provide states for ion localization [11], [12].

On the other hand, the main physical mechanism behind the $\mathrm{BD}$ dynamics of ultra-thin dielectrics $\left(\mathrm{Al}_{2} \mathrm{O}_{3}, \mathrm{HfO}_{2}\right.$, $\mathrm{SiO}_{2}$, and $\mathrm{Si}_{2} \mathrm{~N}_{4}$ ) used in a CMOS technology was recently identified [13], [14]. Briefly, the energy transfer from the $\mathrm{CF}$ to its surroundings promotes the diffusion of the fastest atomic species, which gradually enlarges the $\mathrm{CF}$ connecting the electrodes of the stack causing a progressive increase in the leakage current. Remarkably, SET and BD events show the same statistical behavior [9] and similar micro-structural changes in the dielectric [15]-[19]. Several authors [15]-[17] showed that the BD spot is characterized by the formation of a Si-rich region (for poly- $\mathrm{Si} / \mathrm{SiO}_{x} \mathrm{~N}_{y} / \mathrm{Si}$ stacks) or a metal-rich region (for metal gate/high-K/Si stacks) in the gate dielectric. In this regard, Privitera et al. [18] have shown the presence of metallic species in the oxide for the case of $\mathrm{HfO}_{2}$-based RRAM MIM devices after forming.

In particular, a huge amount of research has been carried out regarding the static $I-V$ characteristics of the RRAMs. However, the time evolution of the switching phenomenon 


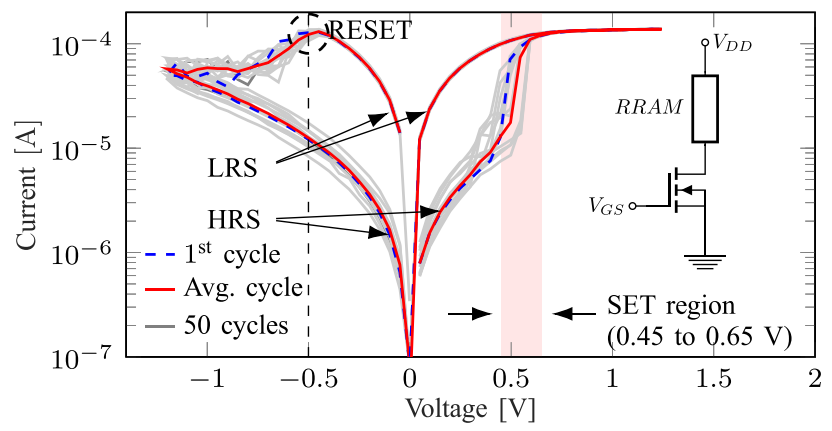

Fig. 1. $\quad-V$ characteristics for the samples under test (50 curves). The red solid line is the average curve, and the blue dashed one stands for the first cycle. The inset in the right side shows the 1T1R assembly, comprising the RRAM structure and its driving nMOS transistor.

is not completely understood yet. In this context, it is clear that despite the recent advances regarding the modeling of the current-voltage behavior of the RS devices [9], [20], more information about the SET event in RRAM devices is needed. Detailed knowledge of the evolution of the CF morphology during forming and SET transitions, as well as the corresponding shape of the $I-V$ curves, is needed to accurately model the switching phenomena and to make reliability predictions. Ultimately, the study of the voltage-time dependence of RS is needed to estimate the minimum time required for writing a bit in an RRAM cell [21]. In this paper, the HRS to LRS transition is studied as a function of time and switching voltage using a large number of measurements. Based on a physical description of the BD dynamics in the dielectrics of common use in the CMOS processes and the aforementioned similarities to the SET event in RRAM, a model for the time evolution of the resistive state of the RRAM as a function of the switching voltage is proposed.

\section{EXPERIMENT}

Experimental data were obtained from $\mathrm{HfO}_{2}$-based RRAM cells. The MIM structure consists of a 10-nm-thick atomiclayer-deposited $\mathrm{HfO}_{2}$ film sandwiched between $\mathrm{Ti}$ and TiN electrodes. The cell is connected in series with an n-type MOSFET, embedding a one-transistor-one-resistor (1T1R) structure, as shown in Fig. 1 (inset). The transistor controls the maximum current that can flow through the memory cell, which in turn determines the resistance window of the device [7], [22]. All the measurements were performed by applying a gate-source voltage $\left(V_{\mathrm{GS}}\right)$ of $1.2 \mathrm{~V}$. Quasi-static $I-V-t$ measurements were performed using a Keithley 4200-SCS equipped with a fast measurement and pulse generator unit (4225-RPM) capable of providing an appropriate time resolution (down to $200 \mathrm{~ns}$ in Fig. 2(b). As reported in a previous work considering similar samples [20], the forming event takes place at $\approx 3.8 \mathrm{~V}$, and the devices exhibit bipolar switching characteristics. Notice that the average SET and RESET voltages are almost symmetric (approximately $\pm 0.5 \mathrm{~V})$. This indicates the voltage-controlled processes for $\mathrm{HfO}_{2}$ in agreement with the previously reported results [23]. Further details about the devices and the experimental setup can be found in [9].
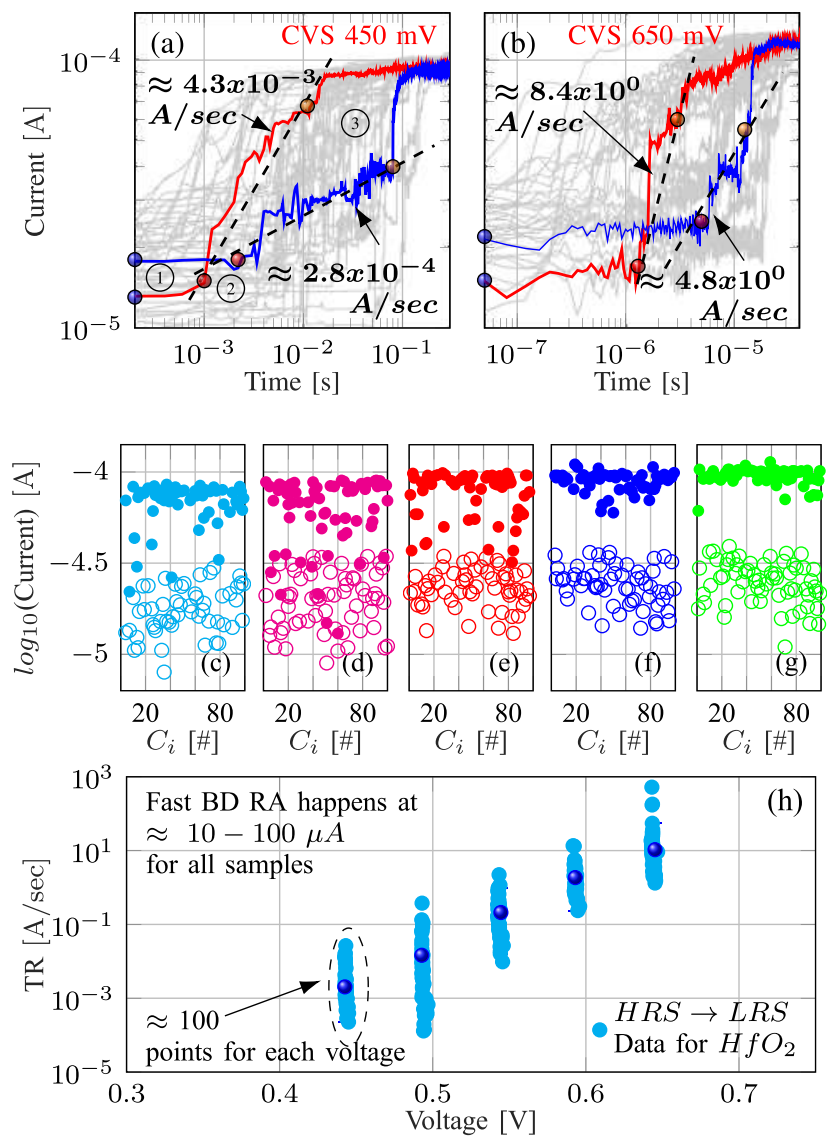

Fig. 2. Current transient of the device under tests under CVS. For clarity, only the lowest $-450 \mathrm{mV}$ - and highest $-650 \mathrm{mV}$ - voltages are shown. Ball markers $1-3$ point out the initial current $\left(l_{\text {init }}\right)$, the onset of the progressive increase of current $\left(I_{o n}\right)$, and the final jump to the compliance level $\left(I_{\text {end }}\right)$, respectively. Initial currents $\left(I_{\text {init }}\right)$ (empty markers) and end currents ( $l_{\text {end }}$ ) (filled markers) at (c) 450, (d) 500, (e) 550, (f) 600, and (g) $650 \mathrm{mV}$. $C_{i}$ in (c)-(g) stands for the cycle number. (h) TR $\left(d /_{\mathrm{Tr}} / d t\right)$ of the samples under test. Approximately, 100 transients and the mean value are reported for each voltage condition $(450,500,550,600$, and $650 \mathrm{mV}$ ). The mean value increases roughly one order of magnitude for every $50 \mathrm{mV}$.

\section{Results}

In order to study the SET event, the whole process, including the wear-out phase and the transition from HRS to LRS, must be explored. Therefore, current-time $(I-t)$ measurements at constant bias were performed using the high-bandwidth setup mentioned in Section II until the fast HRS to LRS transition occurred or in other words, until the current through the device reaches the compliance limit $(\sim 100 \mu \mathrm{A})$. I- $t$ measurements were performed under a constant voltage stress (CVS) of $450,500,550,600$, and $650 \mathrm{mV}$. For clarity, only the transients corresponding to the 450 - and $650-\mathrm{mV}$ stresses are shown in Fig. 2(a) and (b). Such stress voltages were applied in the HRS state and were chosen after the $I-V$ curves shown in Fig. 1, where the HRS to LRS transition occurs for voltages ranging from 0.45 to $0.65 \mathrm{~V}$.

Current through the MIM stack during the HRS to LRS transition $\left(I_{\mathrm{Tr}}\right)$ increases gradually with time [24], evidencing the progressive nature of the SET event [see Fig. 2(a) and (b)]. It is a noisy and progressive process well in agreement with the literature [7], [9], [10], [25] whose duration shows a strong 
voltage dependence and dispersion. Such dispersion will be discussed in Section IV-A. The maximal current growth rate is limited by the bandwidth of the equipment. Fig. 2(c) $-(\mathrm{g})$ shows the initial ( $I_{\text {init }}$, empty symbols) and end ( $I_{\text {end }}$, filled symbols) currents of the acquired transients, including those shown in Fig. 2(a) and (b). It is worth mentioning that the initial current matches the $I-V$ data shown in Fig. 1, where the SET region shows initial currents around $10 \mu \mathrm{A}$. $I_{\text {end }}$ current represents the level from which the acquired transient exhibits a jump to the compliance level (see Fig. 2), leaving the sample in LRS. The time evolution of the HRS to LRS transition is quantified by the slope $d I_{\mathrm{Tr}} / d t$, as defined in [13], [26], and [27]. Such a metric will be subsequently referred to as the transition rate (TR) $[\mathrm{A} / \mathrm{s}]$. The TR values were experimentally evaluated through measurements such as those of Fig. 2(a) and (b), and it is reported for approximately 100 measurements for each voltage value [see Fig. 2(h)]. The comparison between the TR in Fig. 2(a) and (b) and the trend in Fig. 2(h) suggests a strong voltage dependence, as the TR increases almost four orders of magnitude in between these two cases. Similar measurements of the HRS-LRS transition have been previously reported [25], showing a comparable voltage dependence (TR increases as the applied voltage increases).

\section{ANALYSIS AND Discussion}

It has been experimentally shown that both the SET and forming events in RRAM devices [9], [25] and the dielectric BD of gate oxides [13], [14] share some common aspects. Aside from the noisy and progressive increase of the leakage current, whose increase rate depends on the stressing voltage, further insight into these events reveals a few other common points. Previously reported current compliance studies have shown a clear dependence of the $\mathrm{CF}$ characteristics on the maximal current flowing through the device, both for the BD of gate oxides [7] and the SET event in RRAM devices [22]. In addition, transmission electron microscopy imaging of Si-based MOS capacitors prior to and post dielectric $\mathrm{BD}$ [15]-[17] and $\mathrm{HfO}_{2}$-based RRAM cells after forming and cycling [18], [19] shows comparable micro-structural changes in the oxide, suggesting the diffusion of the anodic atomic species into the oxide layer during both events.

Thus, there are not only similar electrical characteristics between the SET event and the gate-oxide BD but also the comparable micro-structural changes and the combination of both points toward a common underlying physical mechanism. In such a scenario, we propose to model the results for the SET event in RRAM devices similar to the gate-oxide BD in MOSFETs. In this regard, Palumbo et al. have presented in [13] a model describing the gate-oxide BD phenomenon in ultra-thin, bulk dielectrics considering $\mathrm{SiO}_{2}$ and various high- $k$ dielectrics with different thermal conductivities deposited on II $I-V$ and Si substrates as well as in 2-D layered materials [28], providing good understanding of the experimental data. Such a model accounts for the progressive nature of the $\mathrm{BD}$ event and quantifies it in terms of $d I_{\mathrm{BD}} / d t$ [namely the degradation rate $(\mathrm{DR}]$, where $I_{\mathrm{BD}}$ is the current through the device during the progressive $\mathrm{BD}$ (PBD). Regarding the physics behind this evolution, the model assumes that the BD process is closely linked to the energy transfer from the $\mathrm{CF}$ itself to its surrounding atomic network. According to this idea, the high temperature associated with the localized current flow (current density of a few $\mathrm{MA} / \mathrm{cm}^{2}$ through a BD spot area of $1-50 \mathrm{~nm}^{2}$ [13], [15], [16]) would promote the electromigration of the fastest available atomic species, thus contributing to the enlargement of the $\mathrm{BD}$ filament connecting the electrodes of the stack. Such electromigration phenomena have been shown to exist in RRAM by Tang et al. [29]. This interpretation of the BD phenomena accounts for the dependence of the $\mathrm{CF}$ features on the current compliance and the presence of the anodic species in the oxide layer after $\mathrm{BD}$, and therefore, it could be used to model the SET event in RRAMs.

It is worth pointing out that the TR shows an evident voltage dependence (four orders of magnitude in $200 \mathrm{mV}$ ) much stronger than the $\mathrm{DR}$ reported for the $\mathrm{BD}$ event $\left(d I_{\mathrm{BD}} / d t\right.$, 3-5 orders of magnitude per volt) [13]. To understand such a big difference, a detailed explanation of the proposed model will be given in the following sections, as well as an explanation about the parameters' change from the gate-oxide BD to the SET event case.

\section{A. Model of the Transition Rate for HRS to LRS as Function of Voltage}

Based on the experimental results and the common underlying physical mechanism that seems to govern both SET and oxide $\mathrm{BD}$, a diffusion phenomenon driven by the energy transfer from the $\mathrm{CF}$ to its surroundings is proposed, including the voltage and oxide thickness dependence. In this context and considering the model reported in [13] for the BD transient, it is possible to express the TR for the HRS to LRS transition as follows:

$$
T R=\frac{d I_{\mathrm{Tr}}}{\mathrm{dt}}=\frac{q V f_{1}}{k_{B} T t_{\mathrm{OX}}^{2}} D I_{\mathrm{SET}}
$$

where $t_{\mathrm{Ox}}$ is the oxide thickness, $T$ is the temperature at the gap in the CF [see Fig. 3(c)], $k_{B}$ is Boltzmann's constant, $D$ is the diffusion constant of the atomic species responsible for the HRS to LRS transition, $V$ is the applied voltage, ISET is the current level at the onset of the HRS to LRS transition (SET event) as expressed in (2), and $f_{1}=n_{e} \lambda_{e} \sigma_{e}$, with being $n_{e}$ the electron density, $\lambda_{e}$ the electron mean free path, and $\sigma_{e}$ the cross section for the electron-atom collision (responsible for the momentum transfer). $f_{1}$ is around the unity value since the defect concentration in the CF is most likely very high [18].

From (1), $d I_{T r} / d t$ is proportional to $D \times I_{\mathrm{SET}}$. This means that the $\mathrm{CF}$ growth rate increases either by increasing $I_{\mathrm{SET}}$, the electron current, or the dominant diffusivity $D$. There is also a dependence on $t_{\mathrm{ox}}, V$, and $T$. Therefore, to use (1), we need to model $I_{\text {SET }}$ and $D$. In addition, the effective value of $t_{\mathrm{Ox}}$ as well as the atomic species involved in the HRS to LRS transition will be further discussed in Sections IV-B and IV-C, respectively.

SET processes were shown to be controlled by the device $I-V$ characteristics, which dictates the local temperature [13], [22] and drives the completion and rupture of a 

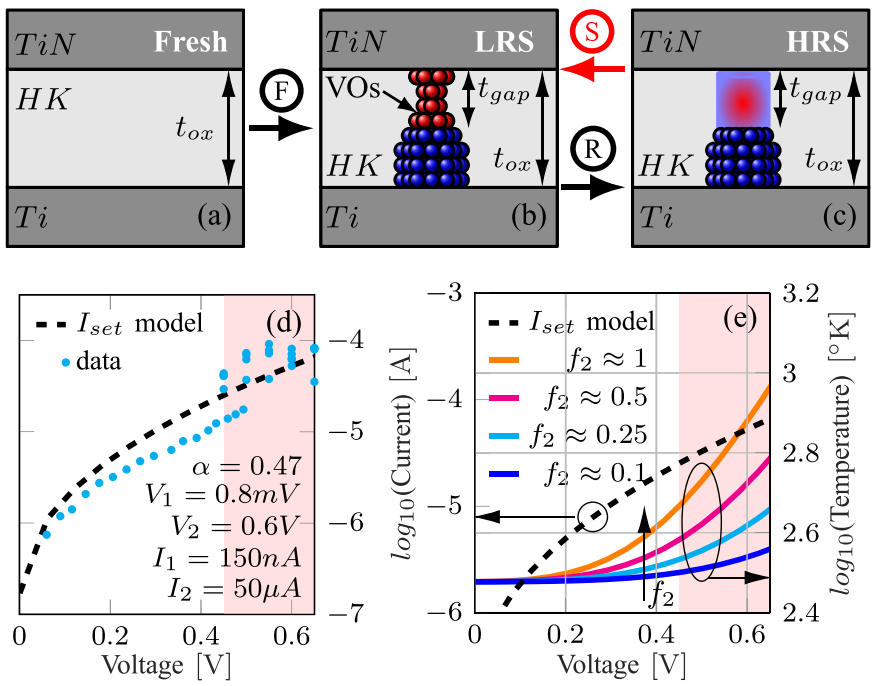

Fig. 3. Representation of the $t_{0 x}$ reduction due to the appearance of a gap in the CF. (a) before forming step. (b) LRS. (c) HRS. Blue balls represent the electrode's atomic species, and the red balls stand for the oxygen vacancies (VOs). (d) ISET model described by (2) versus measurements. (e) Temperature calculations as a function of voltage and the energy loss in the CF constriction. In both (d) and (e) plots, the red shaded zone indicates the voltages employed for the CVS (0.45-0.65 V).

nanoscale CF through the insulating layer [10] [see Fig. 3(b) and (c)]. This accounts for the compliance current used to control the resistance in the SET state [10]. I IET can be described by modeling the discontinuous CF [see Fig. 3(c)] as a quantum size filament with a constriction, according to the Landauer-Buttiker formalism [8], as we have previously reported [7]. However, for simplicity, we have preferred to model the $I_{\mathrm{SET}}-V$ characteristic of the CF in HRS by assuming an interpolation equation [see (2)] [13], [28]. This avoids the use of more parameters. The fitting of our experimental data is shown in Fig. 3(d). $I_{1}, V_{1}$ and $I_{2}, V_{2}$ represent two points in the experimental $I_{\mathrm{SET}}-V$ curve (see HRS curves in Fig. 1) at low and high voltages, respectively, while $\alpha$ is a fitting constant

$$
I_{\mathrm{SET}}=I_{1} \exp \left(\log \left(\frac{I_{2}}{I_{1}}\right)\left(\frac{V-V_{1}}{V_{2}-V_{1}}\right)^{\alpha}\right) .
$$

To model $D$, we first need to model the temperature $T$ in the constriction/discontinuity of the CF. To do so, the heat diffusion equation was solved. We assumed a simplification of spherical symmetry around the CF constriction and considered the source power density to be constant over the inner surface of the sphere. The result is (3), where $f_{2}$ is the fraction of the energy $q V$ per electron lost at the constriction, the dissipated power at the $\mathrm{CF}$ constriction is proportional to $I_{\mathrm{SET}} \times V, T_{\mathrm{amb}}$ is the room temperature, and $k$ is the thermal conductivity of the dielectric

$$
T=\frac{f_{2} V I_{\mathrm{SET}}}{2 \pi t_{\mathrm{ox}} k}+T_{\mathrm{amb}} .
$$

The assumption of power dissipation taking place inside the dielectric layer is reasonable. It has been shown by Takagi et al. [30] for the case of gate-oxide BD in MOSFETs that electrons tunneling through defects responsible for stress-induced leakage current (SILC) in thin oxynitrides do lose a large fraction of their energy in the oxide, which suggests an inelastic process. This behavior has been explained to be a consequence of defect relaxation [31] and was recently confirmed by Lombardo et al. [32] for ultra-thin MOS devices.

$f_{2}$ represents the fraction of energy $q V$ lost by the carriers injected into the dielectric, which ranges from 0 to 1 . While at a large applied voltage, $f_{2}$ tends to 1 in agreement with the electron's high energy loss, and for low voltages, $f_{2}$ decreases, tending to zero for $V=0 . f_{2}$ also depends on the temperature mainly because of phonon-electron scattering [32]. Therefore, $f_{2}$ is a function of voltage and temperature whose behavior is found by the best fit procedure. The influence of $f_{2}$ on the temperature is shown in Fig. 3(e) for different $f_{2}$ values.

Once $T$ at the CF constriction is estimated, then the diffusivity $D$ can be modeled by the exponential law given by (4), where $D_{0}$ is a pre-exponential term and $E_{\text {act }}$ is the diffusion activation energy. It is worth noting that as shown by Lombardo et al. [32], substituting (4) and (3) into (1), an exponential dependence of TR on $f_{2}$ shows off. This may be helpful to explain the remarkable dispersion shown in Fig. 2(a) and (b). If, for example, $f_{2}$ has a $20 \%$ dispersion, then the TR may present a standard deviation of up to two orders of magnitude

$$
D=D_{0} e^{\frac{-E_{\text {act }}}{k_{B} T}} .
$$

\section{B. Effect of the $\mathrm{t}_{\mathrm{ox}}$ Reduction After the Forming Step}

To implement this model for the SET event, the thickness of the dielectric needs to be considered in detail. In a first step, the $\mathrm{CF}$ is created through the fresh insulating layer $\left[\mathrm{HfO}_{2}\right.$ in Fig. 3(a)] by a forming operation [a controlled dielectric BD, see Fig. 3(b)]. Then, the switching mechanism relies on the creation of a gap (RESET) and the restoration of the CF (SET). The width $\left(t_{\mathrm{gap}}\right)$ of such a gap is independent of the oxide layer thickness $\left(t_{\mathrm{ox}}\right)$ [see Fig. 3(c)] but dependent on the current compliance used during the SET event [11], [12], [15].

In a dielectric layer, BD is attributed to the formation of a percolative path between anode and cathode due to the generation of defects of size of $\sim 1 \mathrm{~nm}$ [15], and it is statistically described using the Weibull distribution, an extremevalue distribution appropriate for a weakest-link problem, such as dielectric BD . Being the probability of forming such a path throughout the oxide bulk dependent of the oxide thickness [34], it is possible to use the statistics of the SET switching time $\left(t_{s}\right)$ (i.e., the time to complete the HRS to LRS transition) under constant bias to estimate the effective thickness of the oxide layer after the forming event ( $\left.t_{\text {gap }}\right)$. Fig. 4(a) shows the Weibull plots for $t_{s}$, where the slope (marked as $\beta$ and constant throughout all the voltages considered) is $\sim 1.2$. This suggests a $\mathrm{HfO}_{2}$ film of $\sim 2 \mathrm{~nm}$ from its comparison with the literature [34], [35]. From the model viewpoint, this can be regarded in (1) as a reduction in the physical thickness $t_{\mathrm{ox}}$, as shown in Fig. 3(a) and 3(c). From (3), it is clear that such $t_{\text {ox }}$ reduction causes a significant increase in the temperature at the $\mathrm{CF}$ constriction, as shown in Fig. 4(b) by the dashed and solid lines for the case of $t_{\mathrm{ox}} \approx 10 \mathrm{~nm}$ (fresh device) and $t_{\mathrm{ox}} \approx t_{\mathrm{gap}} \approx 2 \mathrm{~nm}$. It is worth 


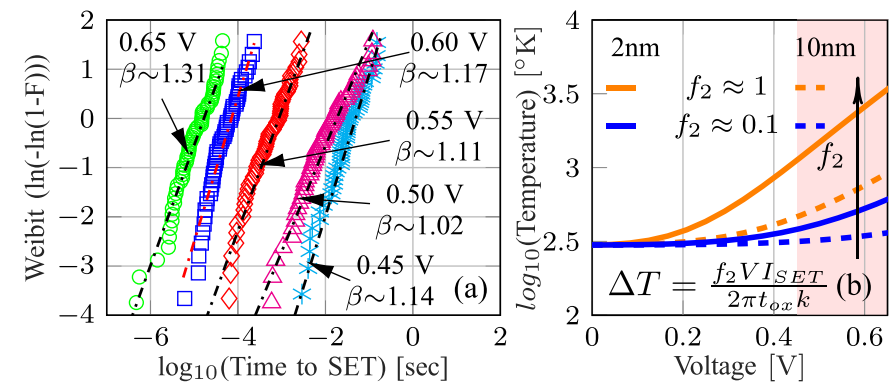

Fig. 4. (a) $t_{s}$ distributions for CVS experiments for $\sim 100$ transitions for each voltage. Symbols are experimental data. Dashed-dotted lines are Weibull fitting results. (b) Temperature in the CF constriction versus the stressing voltage, the resulting $t_{\mathrm{ox}}$, and the energy loss; the red shaded zone indicates the stressing voltage range $(0.45-0.65 \mathrm{~V})$.

mentioning that the local reduction of the thickness caused by the creation of a metal-rich region in the oxide has been observed using cross-sectional high resolution transmission electron microscopy [17], [18], [36].

\section{Increase in the Diffusivity of the Species Involved}

In the model proposed by Palumbo et al. in [13], the creation and growth of the $\mathrm{CF}$ connecting anode and cathode (PBD) are due to the diffusion of the anode/cathode atomic species into the gate dielectric, creating a metallic filamentary path. This has been shown [13] by fitting the DR observed in both $\mathrm{SiO}_{2}$ - and high- $k$ stacks with (1) and evaluating the required diffusivity $D$ as a function of temperature with (4), as shown in Fig. 5 (see curves A, B, and C). Although quite large (of the order of $10^{-13} \mathrm{~cm}^{2} / \mathrm{s}$ at $1000{ }^{\circ} \mathrm{K}$, with low activation energies ranging from 0.7 to $0.3 \mathrm{eV}$ ), such values are in a range compatible with the diffusivity of metals in dielectrics for conditions similar to those adopted here (e.g., the case of $\mathrm{Cu}$ diffusion into $\mathrm{SiO}_{2}$ layers [33]).

In the same way, the diffusivity required to fit the TR versus voltage data has been calculated with (1)-(4) assuming the same values for the fitting parameters [13] ( $E_{\text {act }}$ ranging from 0.3 to $0.7 \mathrm{eV}, f_{2} \sim 0.1$, and $f_{1} \sim 1$ ) and is also plotted against the temperature in Fig. 5 (curve D). The obtained value for $D$ is quite larger $\left(\sim 10^{-6} \mathrm{~cm}^{2} / \mathrm{s}\right.$ at $\left.1000{ }^{\circ} \mathrm{K}\right)$ than the fit diffusivities for the case of gate-oxide $\mathrm{BD}$. It should be mentioned that even in an unlikely scenario in which $100 \%$ of the electron's energy is lost at the BD spot (i.e., $f_{2}=1$ ), the required diffusivity to fit the TR data is significantly higher than the diffusivity obtained from fitting the PBD of gate oxides (for clarity, such fitting results are not shown).

At this point, it is worth recalling that the transition from HRS to LRS has been explained as the vanishing of the gap in the filamentary path. During the forming operation, the CF is jointly created by the migration of the conductive species from the electrodes (blue balls) into the dielectric and the dissociation of oxygen ions $\left(\mathrm{O}^{2-}\right)$, which drift to the top electrode (TE) generating positively charged VOs (red balls) in the oxide layer and a reservoir of $\mathrm{O}^{2-}$ ions in the TE [see Fig. 3(a) and (b)] [12], [38]. Then, the RESET process opens a gap in the CF due to the recombination of the VOs in the $\mathrm{CF}$ with the $\mathrm{O}^{2-}$ ions, which diffuses back from the TE into the oxide [see Fig. 3(c)]. The sample is then in

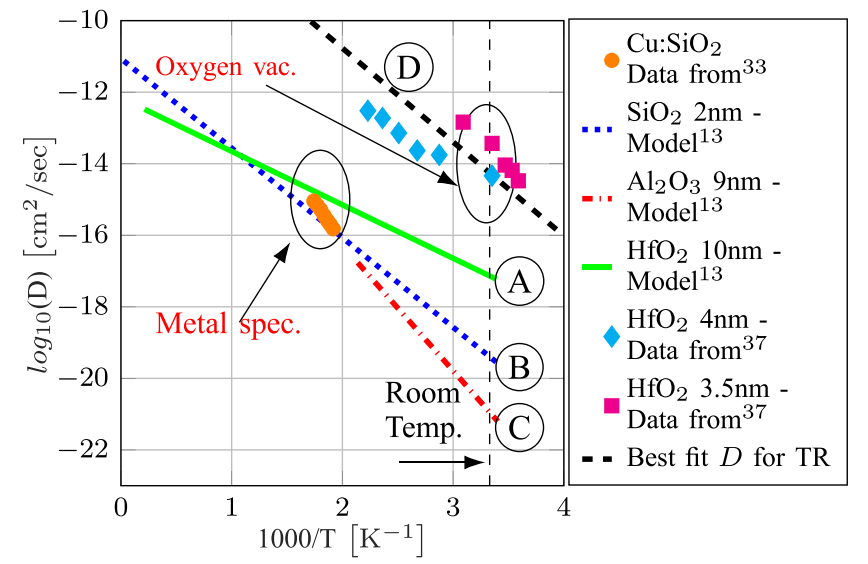

Fig. 5. Diffusivity of the dominant atomic species in the CF versus the reciprocal of temperature. VOs diffusivity [37] is $\sim 10^{4}$ times higher than for the metallic species [13]. $E_{\text {act }}$ ranges from 0.3 to $0.7 \mathrm{eV}$. The diffusivity $D$ required for fitting the TR results is shown to be in the same range as the VOs diffusivity.

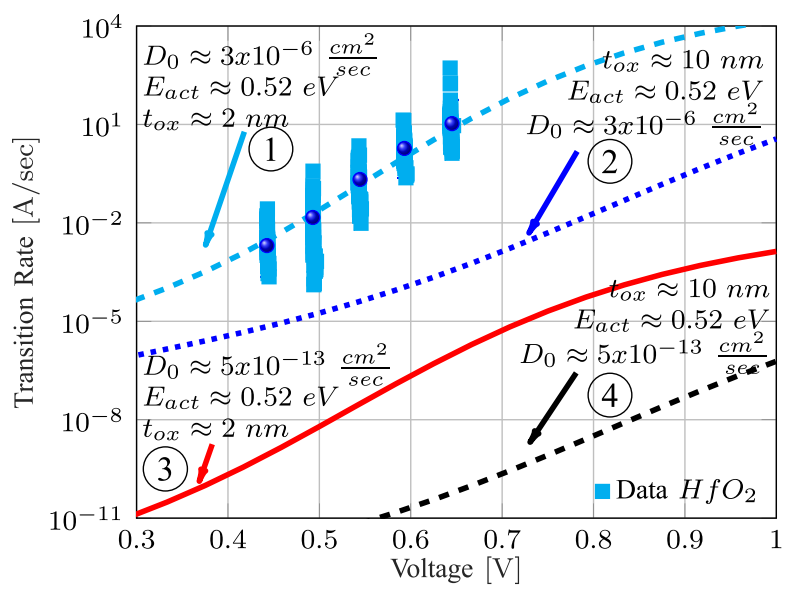

Fig. 6. TR data (square markers-ball markers stand for the mean value-) fit assuming VOs and $t_{\text {gap }}=t_{\text {ox }}$ (cyan dashed line-curve $n^{\circ} 1$ ). In addition, the TR assuming the values reported in the literature for $t_{\mathrm{ox}}$ and $D$ is plotted-curves $n^{\circ} 2,3$, and 4 . The TR increases almost one order of magnitude for every $50-\mathrm{mV}$ step, with a mean value of $2 \times 10^{-3}$, $1.4 \times 10^{-2}, 2.1 \times 10^{-1}, 1.8 \times 10^{0}$, and $1 \times 10^{1} \mathrm{~A} / \mathrm{sec}$ for $450,500,550$, 600 , and $650 \mathrm{mV}$, respectively.

HRS, with a ruptured CF that consists mainly of the anodic atomic species (blue balls) that diffused during the forming. Under such a scheme, the SET event is explained as the completion of the gap due to the migration of $\mathrm{O}^{2-}$ ions toward the TE through a field-assisted and thermally activated effect, which creates the VOs that fill the gap within the CF [see Fig. 3(b)] [20], [38], [39]. This is quite a relevant point to notice, as the diffusivity of VOs, or equivalently the $\mathrm{O}^{2-}$ ions, in a $\mathrm{HfO}_{2}$ layer of thickness similar to $t_{\text {gap }}$ spreads over a range similar to the fit diffusivity for the TR [37] (see curve D in Fig. 5). Therefore, it might, altogether with the mentioned "reduction" of $t_{\mathrm{Ox}}$, explain the higher TR for these samples.

\section{Fitting Results}

The fitting results for the TR as a function of the applied voltage obtained with the proposed model in (1)-(4) are shown in Fig. 6 (see curve $n^{\circ} 1$ ). These results have been superimposed to the scatter plot of the experimental TR data (see the 
square symbols), showing good agreement with the mean value for each voltage (indicated by the ball markers). The proposed fit accounts for both the $t_{\mathrm{Ox}}$ reduction $\left(t_{\mathrm{Ox}}\right.$ considered is equal to $t_{\text {gap }} \sim 2 \mathrm{~nm}$ ) and the increase in diffusivity ( $D_{0}$ is in the order of $\sim 10^{-6} \mathrm{~cm}^{2} / \mathrm{s}$, as other species are considered to complete the $\mathrm{CF}$, i.e., VOs). The rest of the parameters involved remain as previously mentioned $\left(E_{\text {act }} \sim 0.3-0.7 \mathrm{eV}\right.$, $f_{2} \sim 0.1$, and $f_{1} \sim 1$ ).

In order to make clear the impact of both the $t_{\mathrm{ox}}$ reduction and the diffusivity increase $\left(D_{0}\right)$, alternative fitting values are used to plot curves $n^{\circ} 2, n^{\circ} 3$, and $n^{\circ} 4$. Curve $n^{\circ} 2$ shows the TR as a function of voltage assuming fixed $t_{\mathrm{Ox}}$ to the nominal oxide thickness $\left(t_{\mathrm{ox}} \sim 10 \mathrm{~nm}\right)$ but an increase in the atomic species diffusivity $\left(D_{0} \sim 10^{-6} \mathrm{~cm}^{2} / \mathrm{s}\right)$. Curve $\mathrm{n}^{\circ} 3$ presents the resulting curve when only the $t_{\mathrm{Ox}}$ reduction is considered (i.e., $t_{\mathrm{ox}} \sim t_{\text {gap }} \sim 2 \mathrm{~nm}$ and $D_{0}$ corresponding to the metal diffusion in oxides $\sim 10^{-13} \mathrm{~cm}^{2} / \mathrm{s}$ ). Finally, curve $n^{\circ} 4$ presents the TR as a function of the applied voltage considering the same parameter values as in the gate-oxide BD transients, i.e., the diffusivity of metals in oxide layers $\left(D_{0} \sim 10^{-13} \mathrm{~cm}^{2} / \mathrm{s}\right)$ and no $t_{\mathrm{ox}}$ reduction $\left(t_{\mathrm{ox}} \approx 10 \mathrm{~nm}\right)$. This coincides with the DR for gate-oxide BD.

The comparison of curves $n^{\circ} 1$ and $n^{\circ} 4$ evidences that the TR is significantly higher than the DR expected for the voltage range considered. It is also worth noticing that the TR calculated considering only a $t_{\mathrm{Ox}}$ reduction or an increase in diffusivity $\left(D_{0}\right)$ cannot meet the experimental data (see curve $n^{\circ} 1$ versus curve $n^{\circ} 3$ and curve $n^{\circ} 1$ versus curve $n^{\circ} 2$, respectively). This suggests that the $\mathrm{TR}$ as a function of voltage is jointly determined by a combination of both effects, as none of them can fit the results independently.

\section{CONCLUSION}

In this paper, we showed that the HRS to LRS transition (SET) in $\mathrm{HfO}_{2}$-based MIM stacks acting as resistive random access memories (RRAMs) is a progressive phenomenon whose voltage dependence can be modeled in a similar fashion to the PBD of thin oxides [13] by considering a proper adjustment of parameters. In this regard, the time required for the SET transition is studied as a function of the switching voltage considering CVS. As well as in the case of PBD, we propose that the HRS to LRS transition is due to the energy transfer from a CF to its surroundings, which promotes the electromigration of the fastest available atomic species, enlarging the CF. Although the magnitude and voltage acceleration of DR during PBD (forming process) and TR during the SET event are different, in the framework of the model proposed in this paper, such differences are explained as a consequence of an increase in the diffusivity and a reduction of the effective oxide thickness. Diffusivity increases, as the HRS to LRS transition (caused by the completion of the $\mathrm{CF}$ ) is due to the migration of VOs, whose diffusion coefficient is higher than that from electrode's atoms that diffuse during PBD. Second, the reduction in the effective value of $t_{\mathrm{Ox}}$ should be considered, as the SET event takes place in a previously degraded oxide layer, and this increases the speed of the transition. The model presented here accounts for such effects and is in agreement with the previous results reported [18], [20], [38], which presented experimental evidence of both the $t_{\mathrm{ox}}$ reduction and the influence of the VOs.

Finally, it is worth mentioning that, by relating the TR with the temperature and thermal conductivity of the dielectric considered, the TR could be potentially sped up by increasing the temperature at the $\mathrm{CF}$, whether by increasing the SET voltage or by using a material with lower thermal conductivity [40]. As the reduction in the supply voltage is common in current CMOS processes, the second option could eventually lead to shorter writing times for the RRAM devices, which have been proposed as future non-volatile memories.

\section{ACKNOWLEDGMENT}

E. Miranda would like to thank C. Cagli for data provision and discussion.

\section{References}

[1] R. Waser, R. Dittmann, G. Staikov, and K. Szot, "Redox-based resistive switching memories-Nanoionic mechanisms, prospects, and challenges," Adv. Mater., vol. 21, nos. 25-26, pp. 2632-2663, Jul. 2009. doi: 10.1002/adma.200900375.

[2] Y. Shi et al., "Electronic synapses made of layered two-dimensional materials," Nature Electron., vol. 1, no. 8, pp. 458-465, Aug. 2018. doi: 10.1038/s41928-018-0118-9.

[3] D. Ielmini, R. Bruchhaus, and R. Waser, "Thermochemical resistive switching: materials, mechanisms, and scaling projections," Phase Transitions, vol. 84, no. 7, pp. 570-602, Jul. 2011. doi: 10.1080/ 01411594.2011.561478.

[4] J. Park et al., "Resistive switching characteristics of ultra-thin $\mathrm{TiO}_{x}$," Microelectron. Eng., vol. 88, no. 7, pp. 1136-1139, Jul. 2011. doi: 10.1016/J.MEE.2011.03.050.

[5] G. Bersuker et al., "Metal oxide resistive memory switching mechanism based on conductive filament properties," J. Appl. Phys., vol. 110, no. 12, Dec. 2011, Art. no. 124518. doi: 10.1063/1.3671565.

[6] L. Zhang et al., "Unipolar $\mathrm{TaO}_{x}$-based resistive change memory realized with electrode engineering," IEEE Electron Device Lett., vol. 31, no. 9, pp. 966-968, Sep. 2010. doi: 10.1109/LED.2010.2052091.

[7] F. Palumbo, E. Miranda, G. Ghibaudo, and V. Jousseaume, "Formation and characterization of filamentary current paths in $\mathrm{HfO}_{2}$-based resistive switching structures," IEEE Electron Device Lett., vol. 33, no. 7, pp. 1057-1059, Jul. 2012. doi: 10.1109/led.2012.2194689.

[8] E. Miranda and J. Suñé, "Electron transport through broken down ultrathin $\mathrm{SiO}_{2}$ layers in MOS devices," Microelectron. Rel., vol. 44, no. 1, pp. 1-23, 2004. doi: 10.1016/j.microrel.2003.08.005.

[9] A. Rodriguez-Fernandez, C. Cagli, L. Perniola, J. Suñé, and E. Miranda, "Identification of the generation/rupture mechanism of filamentary conductive paths in ReRAM devices using oxide failure analysis," Microelectron. Rel., vols. 76-77, pp. 178-183, Sep. 2017. doi: 10.1016/J.MICROREL.2017.06.088.

[10] D. Ielmini, "Modeling the universal set/reset characteristics of bipolar RRAM by field- and temperature-driven filament growth," IEEE Trans. Electron Devices, vol. 58, no. 12, pp. 4309-4317, Dec. 2011. doi: 10.1109/TED.2011.2167513.

[11] S. Larentis, F. Nardi, S. Balatti, D. C. Gilmer, and D. Ielmini, "Resistive switching by voltage-driven ion migration in bipolar RRAM-Part II: Modeling," IEEE Trans. Electron Devices, vol. 59, no. 9, pp. 2468-2475, Sep. 2012. doi: 10.1109/TED.2012.2202320.

[12] F. Nardi, S. Larentis, S. Balatti, D. C. Gilmer, and D. Ielmini, "Resistive switching by voltage-driven ion migration in bipolar RRAM-Part I: Experimental study," IEEE Trans. Electron Devices, vol. 59, no. 9, pp. 2461-2467, Sep. 2012. doi: 10.1109/TED.2012.2202319.

[13] F. Palumbo, S. Lombardo, and M. Eizenberg, "Physical mechanism of progressive breakdown in gate oxides," J. Appl. Phys., vol. 115, no. 22, 2014, Art. no. 224101. doi: 10.1063/1.4882116.

[14] F. Palumbo, P. Shekhter, K. C. Weinfeld, and M. Eizenberg, "Characteristics of the dynamics of breakdown filaments in $\mathrm{Al}_{2} \mathrm{O}_{3} / \mathrm{InGaAs}$ stacks," Appl. Phys. Lett., vol. 107, no. 12, Sep. 2015, Art. no. 122901. doi: 10.1063/1.4931496. 
[15] S. Lombardo, J. H. Stathis, B. P. Linder, K. L. Pey, F. Palumbo, and C. H. Tung, "Dielectric breakdown mechanisms in gate oxides," J. Appl. Phys., vol. 98, no. 12, 2005, Art. no. 121301. doi: 10.1063/1.2147714.

[16] C. H. Tung et al., "Percolation path and dielectric-breakdown-inducedepitaxy evolution during ultrathin gate dielectric breakdown transient," Appl. Phys. Lett., vol. 83, no. 11, pp. 2223-2225, Sep. 2003. doi: $10.1063 / 1.1611649$.

[17] F. Palumbo, G. Condorelli, S. Lombardo, K. L. Pey, C. H. Tung, and L. J. Tang, "Structure of the oxide damage under progressive breakdown," Microelectron. Rel., vol. 45, nos. 5-6, pp. 845-848, May 2005. doi: 10.1016/J.MICROREL.2004.11.034.

[18] S. Privitera et al., "Microscopy study of the conductive filament in $\mathrm{HfO}_{2}$ resistive switching memory devices," Microelectron. Eng., vol. 109, pp. 75-78, Sep. 2013. doi: 10.1016/J.MEE.2013.03.145.

[19] H. Du et al., "Nanosized conducting filaments formed by atomicscale defects in redox-based resistive switching memories," Chem. Mater, vol. 29, no. 7, pp. 3164-3173, Apr. 2017. doi: 10.1021/ acs.chemmater.7b00220.

[20] A. Rodriguez-Fernandez, C. Cagli, J. Suñe, and E. Miranda, "Switching voltage and time statistics of filamentary conductive paths in $\mathrm{HfO}_{2}$-based ReRAM devices," IEEE Electron Device Lett., vol. 39, no. 5, pp. 656-659, May 2018. doi: 10.1109/LED.2018. 2822047.

[21] S.-S. Sheu et al., "Fast-write resistive RAM (RRAM) for embedded applications," IEEE Design Test Comput., vol. 28, no. 1, pp. 64-71, Jan./Feb. 2011. doi: 10.1109/MDT.2010.96.

[22] D. Ielmini, "Resistive switching memories based on metal oxides: Mechanisms, reliability and scaling," Semicond. Sci. Technol., vol. 31, no. 6, p. 063002, May 2016. doi: 10.1088/0268-1242/31/6/063002.

[23] A. Fantini et al., "Intrinsic switching behavior in $\mathrm{HfO}_{2}$ RRAM by fast electrical measurements on novel $2 \mathrm{R}$ test structures," in Proc. 4th IEEE Int. Memory Workshop, May 2012, pp. 1-4. doi: 10.1109/ IMW.2012.6213646.

[24] B. P. Linder, S. Lombardo, J. H. Stathis, A. Vayshenker, and D. J. Frank, "Voltage dependence of hard breakdown growth and the reliability implication in thin dielectrics," IEEE Electron Device Lett., vol. 23, no. 11, pp. 661-663, Nov. 2002. doi: 10.1109/LED.2002.805010.

[25] Y. Nishi, K. Fleck, U. Böttger, R. Waser, and S. Menzel, "Effect of RESET voltage on distribution of SET switching time of bipolar resistive switching in a tantalum oxide thin film," IEEE Trans. Electron Devices, vol. 62, no. 5, pp. 1561-1567, May 2015. doi: 10.1109/ TED.2015.2411748.

[26] S. Pazos, F. L. Aguirre, E. Miranda, S. Lombardo, and F. Palumbo, "Comparative study of the breakdown transients of thin $\mathrm{Al}_{2} \mathrm{O}_{3}$ and $\mathrm{HfO}_{2}$ films in MIM structures and their connection with the thermal properties of materials," J. Appl. Phys., vol. 121, no. 9, Mar. 2017, Art. no. 094102. doi: $10.1063 / 1.4977851$
[27] R. Pagano et al., "A novel approach to characterization of progressive breakdown in high-k/metal gate stacks," Microelectron. Rel., vol. 48, nos. 11-12, pp. 1759-1764, Nov. 2008. doi: 10.1016/ J.MICROREL.2008.07.071.

[28] F. Palumbo et al., "A review on dielectric breakdown in thin dielectrics: silicon dioxide, high- $k$, and layered dielectrics," Adv. Funct. Mater., vol. 29, Apr. 2019, Art. no. 1900657. doi: 10.1002/adfm.201900657.

[29] K. Tang et al., "Distinguishing oxygen vacancy electromigration and conductive filament formation in $\mathrm{TiO}_{2}$ resistance switching using liquid electrolyte contacts," Nano Lett., vol. 17, no. 7, pp. 4390-4399, Jul. 2017. doi: 10.1021/acs.nanolett.7b01460.

[30] S. Takagi, N. Yasuda, and A. Toriumi, "Experimental evidence of inelastic tunneling in stress-induced leakage current," IEEE Trans. Electron Devices, vol. 46, no. 2, pp. 335-341, Feb. 1999. doi: $10.1109 / 16.740899$.

[31] P. E. Blöchl and J. H. Stathis, "Hydrogen electrochemistry and stressinduced leakage current in silica," Phys. Rev. Lett., vol. 83, no. 2 , pp. 372-375, Jul. 1999. doi: 10.1103/PhysRevLett.83.372.

[32] S. Lombardo, E. Y. Wu, and J. H. Stathis, "Electron energy dissipation model of gate dielectric progressive breakdown in n- and p-channel field effect transistors," J. Appl. Phys., vol. 122, no. 8, Aug. 2017, Art. no. 085701. doi: 10.1063/1.4985794.

[33] Y. Shacham-Diamand, A. Dedhia, D. Hoffstetter, and W. G. Oldham, "Copper transport in thermal $\mathrm{SiO}_{2}$, , J. Electrochem. Soc., vol. 140, no. 8, pp. 2427-2432, Aug. 1993. doi: 10.1149/1.2220837.

[34] E. Y. Wu, J. H. Stathis, and L.-K. Han, "Ultra-thin oxide reliability for ULSI applications," Semicond. Sci. Technol., vol. 15, no. 5, pp. 425-435, Mar. 2000. doi: 10.1088/0268-1242/15/5/301.

[35] Y. H. Kim et al., "Thickness dependence of Weibull slopes of $\mathrm{HfO}_{2}$ gate dielectrics," IEEE Electron Device Lett., vol. 24, no. 1, pp. 40-42, Jan. 2003. doi: 10.1109/LED.2002.807314.

[36] S. Slesazeck and T. Mikolajick, "Nanoscale resistive switching memory devices: a review," Nanotechnology, vol. 30, no. 35, May 2019, Art. no. 352003. doi: 10.1088/1361-6528/ab2084.

[37] S. Zafar, H. Jagannathan, L. F. Edge, and D. Gupta, "Measurement of oxygen diffusion in nanometer scale $\mathrm{HfO}_{2}$ gate dielectric films," Appl. Phys. Lett., vol. 98, no. 15, Apr. 2011, Art. no. 152903. doi: $10.1063 / 1.3579256$.

[38] P. Huang et al., "A physics-based compact model of metal-oxide-based RRAM DC and AC operations," IEEE Trans. Electron Devices, vol. 60 no. 12, pp. 4090-4097, Dec. 2013. doi: 10.1109/TED.2013.2287755.

[39] M. Bocquet et al., "Robust compact model for bipolar oxide-based resistive switching memories," IEEE Trans. Electron Devices, vol. 61, no. 3, pp. 674-681, Mar. 2014. doi: 10.1109/TED.2013.2296793.

[40] M. A. Villena et al., "An in-depth study of thermal effects in reset transitions in $\mathrm{HfO}_{2}$ based RRAMs," Solid-State Electron., vol. 111, pp. 47-51, Sep. 2015. doi: 10.1016/j.sse.2015.04.008. 